\title{
Radiotherapy for Japanese elderly patients with cervical cancer: preliminary survival outcomes and evaluation of treatment-related toxicity
}

\author{
Kenji Yoshida $\cdot$ Ryohei Sasaki $\cdot$ Hideki Nishimura $\cdot$ Daisuke Miyawaki \\ Tetsuya Kawabe $\cdot$ Yoshiaki Okamoto $\cdot$ Koji Nakabayashi $\cdot$ Shigeki Yoshida • \\ Kazuro Sugimura
}

Received: 5 August 2010/ Accepted: 11 November 2010/Published online: 30 November 2010

(C) The Author(s) 2010. This article is published with open access at Springerlink.com

\begin{abstract}
Purpose To examine the preliminary survival outcomes and treatment-related toxicity for elderly patients with cervical cancer treated with radiotherapy (RT).

Methods Forty patients $\geq 75$ years old with cervical cancer who were treated with RT were evaluated. Of these 40 patients, 25 were classified as FIGO stage I or II and 15 as stage III or IVA. Thirty-five patients were treated with radical RT (RRT), and five were treated with surgery plus adjuvant RT $(\mathrm{S}+\mathrm{ART})$. External beam radiotherapy combined with high-dose-rate intracavitary brachytherapy
\end{abstract}

This study was selected for presentation at the 52nd Annual Meeting of the American Society for Therapeutic Radiology and Oncology (ASTRO 52), October 31-November 4, 2010, in San Diego, CA, USA.

K. Yoshida $(\varangle) \cdot$ R. Sasaki $\cdot$ H. Nishimura $\cdot$ D. Miyawaki Department of Radiation Oncology, Kobe University Graduate School of Medicine, 7-5-2 Kusunokicho, Chuouku, Kobe City, Hyogo 650-0017, Japan

e-mail: kyoshi@med.kobe-u.ac.jp

T. Kawabe

Department of Radiology, Dokkyou Medical University,

Tochigi, Japan

Y. Okamoto

Department of Radiology, Osaka Police Hospital,

Osaka, Japan

K. Nakabayashi $\cdot$ S. Yoshida

Department of Gynecology, Kobe University Graduate School of Medicine, Kobe City, Hyogo, Japan

K. Sugimura

Department of Radiology, Kobe University Graduate School

of Medicine, Kobe City, Hyogo, Japan was performed on 31 patients who were treated with RRT and on 2 patients who were treated with $\mathrm{S}+$ ART because of positive vaginal surgical margins. The patients' median age was 78 years (range 75-89 years). Concurrent chemotherapy (CCT) was performed on five patients (RRT: 3, S + ART: 2).

Results The median follow-up period was 20 months (range 1-85 months). Only one patient could not complete RT. The 3-year overall and disease-specific survival (OS and DSS) rates for all patients were 58 and $80 \%$, respectively. Five patients experienced Grade 3 acute toxicity; two were treated with RRT (2/35), and three were treated with S + ART (3/5, 2 of them with CCT). Two patients experienced Grade 3 late toxicity; one was treated with RRT (1/35, with CCT) and the other was treated with $\mathrm{S}+\mathrm{ART}(1 / 5)$. No Grade 4 or higher toxicity was experienced.

Conclusions RRT for elderly patients with cervical cancer is generally effective and safe, but severe toxicity may occur with more aggressive treatment modalities.

Keywords Cervical cancer - Radiotherapy · Elderly patients $\cdot$ Treatment-related toxicity

\section{Introduction}

The population of elderly people has been rapidly increasing in Japan. According to statements by the Ministry of Health, Labor and Welfare, the average life expectancy for men and women in 2008 was 79 and 86 years old, respectively [1]. In particular, the life expectancy of a Japanese woman is the longest in the world. With an increasingly aged society, the number of elderly patients with various malignancies continues to 
increase. In addition, the number of younger cancer patients has also been increasing due to changes in lifestyle and viral infections. In Japan, malignant neoplasms have the highest mortality rate, surpassing cerebrovascular and heart diseases in 1981.

For cervical cancer, the most commonly afflicted age group is women in their late 30 s to early 40 s; the affliction of young women is usually emphasized [2-4]. However, the incidence of cervical cancer increases again after age 70 , and the mortality rate increases with age. Therefore, the increase in the ratio of elderly patients with cervical cancer must be evaluated, and an appropriate treatment modality should be identified. Surgery and/or radiotherapy (RT) are the radical treatment modalities for cervical cancer. For advanced-stage disease, RT with or without concurrent chemotherapy (CCT) is usually the radical treatment of choice. For early-stage disease, the survival outcomes of surgery and RT are known to be similar [5-8]. Although RT seems to be a less invasive treatment, its long-term complications and negative impact on sexual function when compared with surgery are important considerations for younger patients [9-11]. Therefore, there is a trend emerging in which surgery is usually used for younger patients and RT is used for elderly patients. However, although it is obvious that RT plays an important role in the treatment of most stages (I-IVA) of cervical cancer, the recent increase in the elderly population may further increase RT's importance [12, 13].

In this study, we retrospectively analyzed the preliminary survival outcomes and evaluated treatment-related toxicity for Japanese elderly patients ( $\geq 75$ years old) with cervical cancer treated with RT.

\section{Materials and methods}

Patients

At Kobe University Hospital between 2000 and 2009, 40 patients aged 75 or older who had cervical cancer and were treated with RT as the radical or postoperative adjuvant modality were retrospectively evaluated. Patients who received only palliative RT were excluded. Those patients who were followed for $<6$ months, except when this was due to recurrence or death, were also excluded. Between 2000 and 2005, 9 patients were treated, whereas 31 were treated between 2006 and 2009. Clinical staging was performed according to the International Federation of Gynecology and Obstetrics (FIGO) stages [14]. Among the 40 patients, 35 were treated with radical RT (RRT), and 5 were treated with surgery and adjuvant RT $(\mathrm{S}+\mathrm{ART})$. Six patients had pelvic nodal metastases (4 were clinical, 2 were pathological). Thirty-eight tumors were histologically confirmed as squamous cell carcinoma (SCC), and two were confirmed as adenocarcinoma. On the Karnofsky Performance Scale (KPS), 20 patients had scores $>70,17$ had scores between 50 and 70 , and 3 had scores $<50$. Twenty-five patients had stage I or II disease (IA: 1, IB: 4, IIA: 7, IIB: 13), and 15 had stage III or IVA disease (IIIA: 2, IIIB: 11, IVA: 2 ). The median age was 78 years (range 75-89 years). In addition, 29 of the 40 patients had concurrent medical complications. Three patients had a previous history of malignancy (breast cancer, colon cancer, and malignant lymphoma), and one had early-stage lung cancer concurrent with the advanced cervical cancer. Patient information according to clinical factors is shown in Table 1.

Table 1 Patient information according to clinical factors

\begin{tabular}{|c|c|c|}
\hline Factors & Number of patients $($ total $=40)$ & $\%$ \\
\hline \multicolumn{3}{|l|}{ Treatment period } \\
\hline $2000-2005$ & 9 & 22.5 \\
\hline 2006-2009 & 31 & 77.5 \\
\hline \multicolumn{3}{|l|}{ Age (years old) } \\
\hline$\leq 80$ & 27 & 67.5 \\
\hline$>81$ & 13 & 32.5 \\
\hline Median age (range) & $78(75-89)$ & \\
\hline \multicolumn{3}{|c|}{ Karnofsky performance scale score } \\
\hline$>70$ & 20 & 50 \\
\hline $50-70$ & 17 & 42.5 \\
\hline$<50$ & 3 & 7.5 \\
\hline \multicolumn{3}{|l|}{ Stage (FIGO) } \\
\hline IA & 1 & 2.5 \\
\hline IB & 4 & 10 \\
\hline IIA & 7 & 17.5 \\
\hline IIB & 13 & 32.5 \\
\hline IIIA & 2 & 5 \\
\hline IIIB & 11 & 27.5 \\
\hline IVA & 2 & 5 \\
\hline \multicolumn{3}{|l|}{ Histology } \\
\hline SCC & 38 & 95 \\
\hline Adenocarcinoma & 2 & 5 \\
\hline \multicolumn{3}{|l|}{ Nodal metastasis } \\
\hline Yes & 6 & 15 \\
\hline No & 34 & 85 \\
\hline \multicolumn{3}{|l|}{ Medical complications } \\
\hline Yes & 29 & 72.5 \\
\hline No & 11 & 27.5 \\
\hline \multicolumn{3}{|c|}{ History of other cancers } \\
\hline Yes & 4 & 10 \\
\hline No & 36 & 90 \\
\hline
\end{tabular}

SCC squamous cell carcinoma 


\section{Treatment}

In our institution, RRT is recommended as the definitive treatment for patients with cervical cancer $\geq 75$ years old. Surgery is considered if the following criteria are met: young age, high KPS score ( $>70)$, and FIGO I or II. Medical complications and histology (adenocarcinoma) are also important considerations. In addition, the patient's desired treatment choice (RT or surgery) is also considered. Indications for the use of ART are based on pathological findings (nodal metastasis, parametrium invasion, surgical margin, vascular invasion, and/or lymphatic invasion). Based on this institutional guideline, 35 patients were treated with RRT, and the remaining 5 were treated with $\mathrm{S}+$ ART. Among the 35 patients treated with RRT, 31 were treated with external beam radiotherapy (EBRT) combined with high-dose-rate intracavitary brachytherapy (HDR-ICBT), 3 were treated with EBRT alone, and 1 was treated with HDR-ICBT alone. The four patients treated with EBRT or HDR-BT alone had KPS scores $=50$ or less. Of the 31 patients treated with EBRT combined with HDR-ICBT, 2 received boost irradiation for pelvic lymph node metastases. Two of the three patients treated with EBRT alone received boost irradiation for the primary tumor instead of HDR-BT. Among the five patients treated with $\mathrm{S}+\mathrm{ART}$, three received EBRT alone, and two received EBRT combined with HDR-ICBT because of positive vaginal surgical margins. CCT using a platinumbased regimen was performed on five patients. Three were treated with RRT with CCT, and two were treated with $\mathrm{S}+\mathrm{ART}$ with CCT. At our institution, RRT with CCT is performed on younger patients $(<80)$ with high KPS scores $(>70)$ and FIGO IIB or higher. The presence of medical complications is also an important consideration. Based on these criteria, three patients were treated with RRT with CCT. Adjuvant CCT has been performed on patients with multiple pathological risk factors (at least 3) since 2008. Postoperative KPS score $(>70)$ is also considered to be important because $\mathrm{S}+\mathrm{ART}$ with CCT is a very aggressive treatment for elderly patients; two were ultimately treated with this modality. The patient distribution per treatment modality is shown in Table 2.

The patients who received EBRT combined with HDRICBT were initially treated with whole pelvic irradiation using a box field and high-energy $10 \mathrm{MV}$ X-ray photons from a linear accelerator with a daily fraction size of 1.8-2.0 Gy delivered five times per week. A centrally shielded field using anterior/posterior opposed portals was applied just before starting HDR-ICBT. The patients who received EBRT alone were also initially treated with whole pelvic irradiation. A boost to the primary tumor was delivered using a three-dimensional conformal technique, and a pelvic lymph node boost was delivered using the
Table 2 Patient distribution per treatment modality

\begin{tabular}{lll}
\hline & $\begin{array}{l}\text { Number } \\
\text { of patients }\end{array}$ & $\begin{array}{l}\text { Use } \\
\text { of CCT }\end{array}$ \\
\hline RRT & & \\
EBRT + HDR-ICBT (with nodal boost) & $31(2)$ & 3 \\
EBRT (with local boost) & $3(2)$ & 0 \\
HDR-ICBT & 1 & 0 \\
S + ART & 3 & \\
EBRT & 2 & 1 \\
EBRT + ICBT & 2 & 1 \\
\hline
\end{tabular}

$R R T$ radical radiotherapy, EBRT external beam radiotherapy, $H D R-I C B T$ high-dose-rate intracavitary brachytherapy, $S$ surgery, $A R T$ adjuvant radiotherapy, $C C T$ concurrent chemotherapy

anterior/posterior opposed portals. The median total dose of EBRT was 50.4 Gy (range 16.2-61.2 Gy). The HDRICBT was done with a Microselectron HDR (Nucletron, The Netherlands) using a 192-Iridium remote afterloading system at 1-week intervals during the period of EBRT. The median total dose to point A was $20.0 \mathrm{~Gy}$ (range 4.5-31.0 Gy) with a single fraction size of 4.0-6.5 Gy. Treatment planning for HDR-ICBT was performed at each irradiation using PLATO Brachytherapy Planning System version 3.2 (Nucletron, The Netherlands). Evaluation of the rectal and bladder dose was performed according to ICRU Report 38 [15].

Follow-up, evaluation of treatment-related toxicity, and statistical analysis

After completion of their treatment, most patients were followed up by gynecological and radiation oncologists every month during the first year, primarily because elderly patients tolerate RT less well and unexpected toxicity might be experienced. However, patients who lived far from our institution were followed up every 2-3 months. Afterward, follow-up was conducted every 3-6 months to detect recurrence and late toxicity. A gynecological examination was performed, and the tumor marker was checked at every visit. SCC Antigen was used for patients who had SCC, and Carcinoembryonic Antigen (CEA) was usually used for patients who had adenocarcinoma. Radiographic examinations (CT scan or MRI) were performed as necessary.

Both acute and late treatment-related toxicity were evaluated using medical records and CTC-AE version 4.0 [16]. Acute toxicity was defined as those events that occurred within 90 days from the start of the treatment, and late toxicity was defined as those events that either occurred $>90$ days from the start of the treatment or persisted beyond 90 days. 
Statistical analyses were performed using Sigma Plot 9.0 software (Systat Corporation, CA, USA). Survival rates were calculated with the Kaplan-Meier method and compared with the use of log-rank test. The follow-up period was calculated from the start of the treatment. $P$ values $<0.05$ were considered statistically significant.

\section{Results}

Patient status and patterns of failure

The median follow-up period for all patients was 20 months (range 1-85 months). The median follow-up period for survivors was also 20 months (range 6-85 months). Of the initial 40 patients, 38 completed the treatment as planned, 1 completed with a delay due to concomitant heart disease, and 1 could not complete the treatment because of acute toxicity. These two patients who experienced delay or cancellation had lower KPS scores $(<50)$. Seven patients experienced recurrence: four locally, one in the para-aortic lymph nodes, one distantly, and one with only tumor marker (SCC Antigen) elevation. Even though a thoracic-abdominal contrast enhanced $\mathrm{CT}$ scan, a pelvic MRI, a gynecologic examination and cytology were performed, a recurrent tumor could not be detected at any site. However, this patient was presumed to have microscopic recurrence because SCC Antigen increased continuously. Regarding the clinical stages, one patient was classified as IIA, one as IIIA, and five as IIIB. Six of the seven patients with recurrence were treated with RRT, and one was treated with S + ART. During the period of this study, nine patients died. Among them, five died because of the primary disease, and four died from other causes. The patient who could not complete the treatment had persistent disease and died of the primary disease. Of the remaining two patients who experienced recurrence, one with the para-aortic lymph nodes metastases is alive with the disease and one with tumor marker elevation apparently died from a different cause. The patient who had early-stage lung cancer concurrently with the cervical cancer received the left lower lobe resection after completion of RT. The pathological diagnosis was adenocarcinoma, pT2N0M0. This patient experienced multiple bone metastases (bilateral sacroiliac joints and lumber spine) about 22 months after surgery. Bisphosphonate has been continuously administered, and the patient is doing well without pain.

Preliminary survival outcomes

The 3-year overall and disease-specific survival (OS and DSS) rates for all the patients were 58 and $80 \%$, respectively (Fig. 1a, b). The 3-year OS rate for patients in FIGO stage I or II was $69 \%$, and the rate for stage III or IVA patients was $40 \%(P=0.04)$. The 3 -year DSS rate for patients in FIGO stage I or II was $89 \%$ and that for stage III or IVA patients was $66 \%(P=0.04)$. The patients were also divided into two groups according to age; there were 27 patients aged $\leq 80$ years with a median follow-up of 26 months (range 1-85 months) and 13 patients aged $>80$ years with a median follow up of 14 months (range $7-61$ months). The 3 -year OS rates for patients aged $\leq 80$ and $>80$ years were 62 and $42 \%$, respectively $(P=0.89)$. The 3 -year DSS rates for patients aged $\leq 80$ and $>80$ years were 75 and $100 \%$, respectively $(P=0.21)$. Survival was also analyzed according to KPS score. The 3 -year OS rates for patients with KPS scores $>70$ and $\leq 70$ were 61 and $55 \%$, respectively $(P=0.15)$. The 3 -year DSS rates for patients with KPS scores $>70$ and $\leq 70$ were 92 and $65 \%$, respectively $(P=0.11)$.

\section{Treatment-related toxicity}

The details regarding acute toxicity are shown in Table 3 . The most common acute toxicity was diarrhea (18/40
Fig. 1 Preliminary overall and disease-specific survival (OS and DSS) rates for all patients ( $n=40)$ using the KaplanMeier method with a median follow-up of 20 months (range 1-85 months). The 3-year OS and DSS rates were $58 \%$ (a) and $80 \%(\mathbf{b})$, respectively
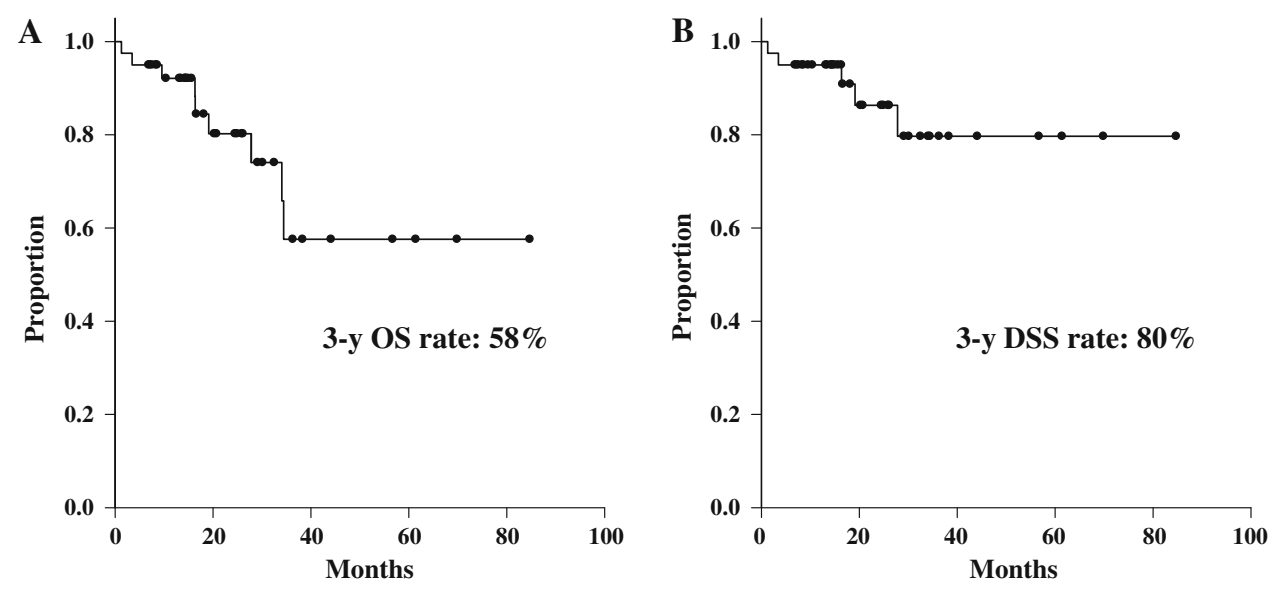
Table 3 Acute treatmentrelated toxicity according to treatment modality (CTC-AE version 4.0)

\begin{tabular}{|c|c|c|}
\hline & RRT (use of CCT), total: 35 & $\mathrm{~S}+$ ART (use of CCT), total: 5 \\
\hline \multicolumn{3}{|c|}{ Gastrointestinal } \\
\hline Grade $1-2$ & $\begin{array}{l}\text { Diarrhea: } 16(2) \text {, gastrointestinal } \\
\text { pain: } 2\end{array}$ & Diarrhea: 1(1) \\
\hline Grade 3 & Diarrhea: 1 & $\begin{array}{l}\text { Intestinal infection: } 1(1)^{\mathrm{a}} \text {, } \\
\text { intestinal obstruction: } 1\end{array}$ \\
\hline Grade 4 & 0 & 0 \\
\hline \multicolumn{3}{|l|}{ Genitourinary } \\
\hline Grade $1-2$ & Urinary frequency: 3 , cystitis: 1 & Urinary tract obstruction: 1 \\
\hline Grade 3 & Cystitis: 1 & $\begin{array}{l}\text { Cystitis: } 1 \text {, urinary tract } \\
\text { obstruction: } 1(1)^{\mathrm{a}}\end{array}$ \\
\hline Grade 4 & 0 & 0 \\
\hline
\end{tabular}

$R R T$ radical radiotherapy, $S$ surgery, $A R T$ adjuvant radiotherapy, $C C T$ concurrent chemotherapy

a Same patient
Table 4 Late treatment-related toxicity according to treatment modality (CTC-AE version 4.0)

\begin{tabular}{lll}
\hline & $\begin{array}{l}\text { RRT (use of CCT), } \\
\text { total: 35 }\end{array}$ & $\begin{array}{l}\text { S + ART (use of CCT), } \\
\text { total: 5 }\end{array}$ \\
\hline $\begin{array}{ll}\text { Gastrointestinal } \\
\text { Grade 1-2 }\end{array}$ & Rectal bleeding: 2 & 0 \\
Grade 3 & 0 & Intestinal obstruction \\
Grade 4 & 0 & 0 \\
Genitourinary & & \\
Grade 1-2 & Cystitis: 2 & 0 \\
Grade 3 & Cystitis: 1(1) & 0 \\
Grade 4 & 0 & 0 \\
Other & & \\
Grade 1-2 & Lymphedema: 2 & Lymphedema: 1 \\
Grade 3 & 0 & 0 \\
Grade 4 & 0 & 0
\end{tabular}

$R R T$ radical radiotherapy, $S$ surgery, $A R T$ adjuvant radiotherapy, $C C T$ concurrent chemotherapy restart RT. The patients who experienced cystitis or small intestine infections were managed by the administration of antibiotics and intravenous transfusions without delaying the RT. The urinary tract obstruction was resolved by urological intervention. The small intestine obstruction was managed by conservative treatment, such as fasting, antibiotic administration, and continuous intravenous transfusion. In both the urinary tract and small intestine obstructions, abdominal CT scans were performed immediately after the symptoms occurred, and progressive disease was excluded.

Currently, Grade 3 late toxicity has occurred in two patients (2/40 patients, 5\%). One of these two patients (treated with RRT with CCT) experienced Grade 3 hemorrhagic cystitis. The other patient (treated with $\mathrm{S}+\mathrm{ART}$ ) experienced a Grade 3 acute small intestine obstruction and a Grade 3 late small intestine obstruction. No Grade 4 or greater late toxicity was experienced. The hemorrhagic cystitis was managed by endoscopic hemostasis. The small intestine obstruction was also managed by conservative treatment. Abdominal CT scans were performed in both cases and progressive disease was excluded before starting the toxicity management. The details regarding late toxicity are shown in Table 4.

\section{Discussion}

Choosing a treatment for elderly patients with various malignancies is usually difficult. Careful evaluation of their general condition and concomitant medical problems must be performed before the treatment begins. Compared with young patients, safer and more effective modalities should be chosen because severe toxicity may lead to cancellation or delay of the treatment and subsequent loss of quality of life [17-21]. Generally, RT is thought to be less invasive than surgery or chemotherapy. Moreover, with recent technical developments, a reduction of radiation-related toxicity has been achieved, and the safety of RT is 
increasing markedly. Therefore, RT is usually chosen for elderly patients as a single modality, although sometimes $\mathrm{RT}$ is combined with surgery and/or chemotherapy. Certainly, RT has taken on a greater role in aging societies such as Japan. For example, in this study, just 9 patients were treated from 2000 to 2005 , but 31 were treated from 2006 to 2009.

Although there are several large retrospective studies that have analyzed treatment results and prognostic factors, whether age is a negative prognostic factor remains controversial [12, 13, 22-27]. However, most reports have demonstrated that RT is effective for elderly patients. For example, Ikushima et al. analyzed 727 patients with cervical cancer and reported that the 5- and 10-year diseasespecific survival rates of 132 patients aged $\geq 75$ years were 66 and 57\%, respectively. Thus, age was not a significant prognostic factor in that study [13]. Chen et al. analyzed a total of 295 patients. They reported that the 5 -year cause-specific survival rates of 79 patients aged $\geq 70$ years with respect to FIGO stage were $100 \%$ for IB, $85 \%$ for IIA, $78 \%$ for IIB, and $42 \%$ for III. Thus, again age was not a significant prognostic factor in this case [26]. On the other hand, Brun et al. analyzed a total of 308 patients and reported that the 5-year survival rate of 31 patients aged $\geq 75$ years was $42 \%$ and that age was a significant prognostic factor. However, they also reported that the survival of those over 75 years was not different from that of the rest of the population [23]. Although the median follow-up of our study was shorter and the number of cases is currently smaller, our observed survival rates are reasonable compared with previous reports. Our results also indicate that the clinical stage might have prognostic value in determining survival outcomes, but age did not have prognostic value in such an elderly population. Interestingly, the DSS rate of patients aged $>80$ years was $100 \%$. Whether "slow oncological progression" was associated with this result is unclear because of the small number of patients and the short follow-up period. Therefore, this result cannot be used as evidence for a more limited treatment choice at present. However, RRT alone should be the first choice for patients $>80$ years old. The survival rates of the patients with high KPS scores $(>70)$ were better than those with low KPS scores $(\leq 70)$, but the difference was not significant. KPS was not a significant prognostic factor in this preliminary result, but it may have a large impact on long-term survival. To evaluate survival outcomes accurately and verify prognostic factors such as clinical stage, age, and KPS, more cases need to be analyzed, a longer follow-up period is needed, and the results need to be compared with those of a younger population. Finally, the most appropriate treatment choice for elderly patients should be established.
Both acute and late toxicity should be evaluated carefully to establish a safe modality that achieves better survival outcomes and preserves the quality of life of elderly patients with cervical cancer. Lindegaard et al. reported that treatment was completed as planned in $68 \%$ of cases, delayed in $29 \%$ of cases, and stopped prematurely in $3 \%$ of cases. They concluded that elderly patients with cervical cancer in otherwise good health may tolerate radical radiotherapy with acceptable toxicity and reasonable survival rates [28]. In our study, 38 of 40 (95\%) patients completed the treatment as planned; $1(2.5 \%)$ completed after a delay and $1(2.5 \%)$ could not complete the treatment. The two patients who experienced delay or cancellation of the treatment had KPS scores $<50$ and had RRT performed, but they could not receive HDR-BT. This result also indicates that elderly patients in good health can tolerate RRT (EBRT combined with HDR-ICBT). However, those with a poor performance status should be treated carefully; in some instances, a less invasive RRT (EBRT alone) must be chosen. For elderly patients in good health, tolerance for more aggressive treatment modalities such as RRT with CCT or S + ART with or without CCT should be discussed carefully. In our study, 8 patients with KPS scores $>70$ were treated with these more aggressive modalities (RRT with CCT: 3, S + ART: 3, S + ART with CCT: 2). As described above, the indications for the use of these aggressive modalities involved age, KPS, FIGO stage, and pathological risk factors. Regarding the patients treated with RRT with CCT, all of them were $<80$ years old and had KPS scores $>70$. Two of them were FIGO IIB and the remaining one was IIIB. Regarding the patients treated with $\mathrm{S}+$ ART with or without CCT, 4 were 75 , and 1 was 76 years old. All of them had KPS scores $>70$ and had stage II disease (IIA: 2, IIB: 3). Adjuvant CCT has been performed on patients with postoperative KPS scores $>70$ and multiple pathological risk factors (at least 3 ) since 2008. As a result, two were treated with $\mathrm{S}+\mathrm{ART}$ with CCT. One had wide parametrium invasion and both vascular and lymphatic invasion. The other had a large tumor $(>4 \mathrm{~cm})$, parametrium invasion, vascular invasion, and a positive vaginal surgical margin. Although nodal metastasis was the most important prognostic factor, two patients who had pathological nodal metastasis did not receive adjuvant CCT. This was because one had postoperative KPS score $=50$, whereas the other was one of the oldest patient treated in 2000 and adjuvant CCT was not performed for elderly patients at that time. Therefore, they were treated with S + ART without CCT. All of the patients treated with these aggressive modalities completed the treatment without delay, but three of them (37.5\%) experienced Grade 3 acute toxicity during and soon after the completion of RT. These results indicate that these aggressive modalities are not always safe in terms of 
acute toxicity. As for late toxicity, although the median follow-up was shorter, Grade 3 late toxicity was experienced by 2 of $40(5 \%)$ patients, and no Grade 4 or higher late toxicity was experienced in our study. Several authors reported that the occurrence rates of Grade 3 or greater late morbidities were less than approximately $10 \%$, and our results are compatible with those of previous reports [12, 13, 28, 29]. However, we should emphasize that Grade 3 late toxicity was only experienced in patients treated with the more aggressive modalities (RRT with CCT: 1, S + ART: 1). Aggressive modalities may be tolerable for patients with a good performance status, but they can easily cause severe acute or late toxicity compared with RRT alone. Considering these results, when aggressive treatment modalities are performed in elderly patients, management of both acute and late toxicity is very important to avoid delay or cancellation and to maintain quality of life. The finding that patients with KPS scores $>70$ can tolerate aggressive modalities with appropriate management, whereas those with KPS scores $<50$ may not tolerate even RRT alone, is also very important. KPS should be considered as one of the determinants in selecting a treatment modality for elderly patients.

In conclusion, the number of elderly patients with cervical cancer is increasing, and RRT provides good survival outcomes with acceptable toxicity. However, indications for the use of more aggressive modalities should be assessed carefully, even for patients who are in quite good health. Therefore, to establish appropriate treatment strategies, including combinations of RT with less invasive surgery and/or chemotherapy, larger studies and prospective studies should be performed. Finally, better survival outcomes and preservation of the quality of life may be achievable for the growing elderly population.

Conflict of interest We declare that we have no conflict of interest.

Open Access This article is distributed under the terms of the Creative Commons Attribution Noncommercial License which permits any noncommercial use, distribution, and reproduction in any medium, provided the original author(s) and source are credited.

\section{References}

1. Abridged life tables for Japan 2008, Ministry of Health, Labour and Welfare. http://www.mhlw.go.jp/english/database/db-hw/vs02. html

2. Schwartz S (2009) Young cervical cancer patients and fertility. Semin Oncol Nurs 25:259-267

3. Yang L, Fujimoto J, Qiu D, Sakamoto N (2009) Trends in cancer mortality in Japanese adolescents and young adults aged 15-29 years, 1970-2006. Ann Oncol 20:758-766
4. Kokawa K, Takekida S, Kamiura S, Kita M, Enomoto T, Kawaguchi R, Saito J, Horie A, Umesaki N (2010) The incidence, treatment and prognosis of cervical carcinoma in young women: a retrospective analysis of 4, 975 cases in Japan. Eur J Gynaecol Oncol 31:37-43

5. Landoni F, Maneo A, Colombo A, Placa F, Milani R, Perego P, Favini G, Ferri L, Mangioni C (1997) Randomised study of radical surgery versus radiotherapy for stage Ib-IIa cervical cancer. Lancet 350:535-540

6. Perez CA, Grigsby PW, Camel HM, Galakatos AE, Mutch D, Lockett MA (1995) Irradiation alone or combined with surgery in stage IB, IIA, and IIB carcinoma of uterine cervix: update of a nonrandomized comparison. Int $\mathrm{J}$ Radiat Oncol Biol Phys 31:703-716

7. Gaze MN, Kelly CG, Dunlop PR, Redpath AT, Kerr GR, Cowie VJ (1992) Stage IB cervical carcinoma: a clinical audit. Br J Radiol 65:1018-1024

8. Yamashita H, Nakagawa K, Tago M, Shiraishi K, Nakamura N, Ohtomo K, Oda K, Nakagawa S, Yasugi T, Taketani Y (2005) Comparison between conventional surgery and radiotherapy for FIGO stage I-II cervical carcinoma: a retrospective Japanese study. Gynecol Oncol 97:834-839

9. Undurraga M, Loubeyre P, Dubuisson JB, Schneider D, Petignat $P$ (2010) Early-stage cervical cancer: is surgery better than radiotherapy? Expert Rev Anticancer Ther 10:451-460 (Review)

10. Korfage IJ, Essink-Bot ML, Mols F, van de Poll-Franse L, Kruitwagen R, van Ballegooijen M (2009) Health-related quality of life in cervical cancer survivors: a population-based survey. Int J Radiat Oncol Biol Phys 73:1501-1509

11. Frumovitz M, Sun CC, Schover LR, Munsell MF, Jhingran A, Wharton JT, Eifel P, Bevers TB, Levenback CF, Gershenson DM, Bodurka DC (2005) Quality of life and sexual functioning in cervical cancer survivors. J Clin Oncol 23:7428-7436

12. Sakurai H, Mitsuhashi N, Takahashi M, Yamakawa M, Akimoto T, Hayakawa K, Niibe H (2000) Radiation therapy for elderly patient with squamous cell carcinoma of the cervix. Gynecol Oncol 77:116-120

13. Ikushima H, Takegawa Y, Osaki K, Furutani S, Yamashita K, Kawanaka T, Kubo A, Kudoh T, Nishitani H (2007) Radiation therapy for cervical cancer in the elderly. Gynecol Oncol 107: 339-343

14. Creasman WT (1995) New gynecologic cancer staging. Gynecol Oncol 58:157-158

15. International Commission on Radiation Units and Measurements (1985) Dose and volume specification for intracavity therapy in gynecology. ICRU report 38. ICRU, Washington

16. Common Toxicity Criteria version 4.0 Japanese TranslationJCOG (2009) Japan Clinical Oncology Group

17. Terret C, Albrand G, Droz JP (2004) Management and geriatric assessment of cancer in the elderly. Clin Prostate Cancer 2:236240

18. Sekine I, Yamamoto N, Kunitoh H, Ohe Y, Tamura T, Kodama T, Saijo N (2004) Treatment of small cell lung cancer in the elderly based on a critical literature review of clinical trials. Cancer Treat Rev 30:359-368

19. Harlacher R, Füsgen I (2000) Geriatric assessment in the elderly cancer patient. J Cancer Res Clin Oncol 126:369-374

20. Yancik R, Havlik RJ, Wesley MN, Ries L, Long S, Rossi WK, Edwards BK (1996) Cancer and comorbidity in older patients: a descriptive profile. Ann Epidemiol 6:399-412

21. Hurria A, Wong FL, Villaluna D, Bhatia S, Chung CT, Mortimer J, Hurvitz S, Naeim A (2008) Role of age and health in treatment recommendations for older adults with breast cancer: the perspective of oncologists and primary care providers. J Clin Oncol 26:5386-5392 
22. Mitchell PA, Waggoner S, Rotmensch J, Mundt AJ (1998) Cervical cancer in the elderly treated with radiation therapy. Gynecol Oncol 71:291-298

23. Brun JL, Stoven-Camou D, Trouette R, Lopez M, Chene G, Hocké C (2003) Survival and prognosis of women with invasive cervical cancer according to age. Gynecol Oncol 91:395-401

24. Wright JD, Gibb RK, Geevarghese S, Powell MA, Herzog TJ, Mutch DG, Grigsby PW, Gao F, Trinkaus KM, Rader JS (2005) Cervical carcinoma in the elderly: an analysis of patterns of care and outcome. Cancer 103:85-91

25. de Rijke JM, van der Putten HW, Lutgens LC, Voogd AC, Kruitwagen RF, van Dijck JA, Schouten LJ (2002) Age-specific differences in treatment and survival of patients with cervical cancer in the southeast of The Netherlands, 1986-1996. Eur J Cancer 38:2041-2047
26. Chen SW, Liang JA, Yang SN, Lin FJ (2003) High dose-rate brachytherapy for elderly patients with uterine cervical cancer. Jpn J Clin Oncol 33:221-228

27. Mitsuhashi N, Takahashi M, Nozaki M, Yamakawa M, Takahashi T, Sakurai H, Maebayashi K, Hayakawa K, Niibe H (1995) Squamous cell carcinoma of the uterine cervix: radiation therapy for patients aged 70 years and older. Radiology 194:141-145

28. Lindegaard JC, Thranov IR, Endelholm SA (2000) Radiotherapy in the management of cervical cancer in elderly patients. Radiother Oncol 56:9-15

29. Magné N, Mancy NC, Chajon E, Duvillard P, Pautier P, Castaigne D, Lhommé C, Morice P, Haie-Meder C (2009) Patterns of care and outcome in elderly cervical cancer patients: a special focus on brachytherapy. Radiother Oncol 91:197-201 